\title{
Healthcare Management: Prospects and Risks of Using Blockchain Technology
}

\author{
${ }^{\nabla} 1$ Li Wenzheng, ${ }^{2}$ Marina Alievna Tappaskhanova, ${ }^{3}$ Galina Nikiporets-Takigawa, ${ }^{4}$ Anna Evgenievna \\ Gorokhova, ${ }^{5}$ Vitaly V. Goncharov \\ ${ }^{1}$ Moscow State University, Moscow, Russia \\ wenzheng.li@mail.ru \\ ${ }^{2}$ N.F. Gamaleya Federal Research Center for Epidemiology \& Microbiology, Moscow, Russia \\ tap-marina@mail.ru \\ ${ }^{3}$ Russian State Social University, Moscow, Russia \\ NikiporetsGIu@rgsu.net \\ ${ }^{4}$ Moscow Polytechnic University, Moscow, Russia \\ a.e.gorokhova@list.ru \\ ${ }^{5}$ Kuban State Agrarian University named after I.T. Trubilin, Krasnodar, Russia \\ niipgergo2009@mail.ru
}

Received: 21st August 2020, Accepted: 14th September 2020, Published: 31st October 2020

\begin{abstract}
Blockchain technology is a fundamental discovery that plays an indispensable part in today's professional world. this technology has even entered many industries and businesses these days. Even the health sector has not been spared from this trend. Blockchain is slowly preparing to enter the medical industry. Over the past five years, global technology giants such as IBM have devoted considerable resources to conduct comprehensive studies on the Chinese bloc. The results of these studies result in greater knowledge of the applications of this technology in the field of health and wellness. The results of a survey show that more than half of health executives are keen to see some form of blockchain technology enter the medical system in the future.

The study grants an overview of appearing blockchain-healthcare technologies and relevant applications, highlighting studies in this area, and demonstrates the blockchain technology's potentials in enhancing the healthcare industry.
\end{abstract}

Keywords

Healthcare Management; Data Management; Internet of Medical Things, Blockchain, Supply Chain Management.

\section{Introduction}

Blockchain is a system for recording data. This data can be, for example, bank transactions or ownership documents, appointments, personal messages or other information. The feature of blockchain is that the storage of this data is possible without a central manager and owner, and the stored data cannot be distorted or destroyed by destroying a central point. The most popular use of blockchain is the Bitcoin currency code[1].

Blockchain makes it easier to secure online activities. It is a decentralized and shared digital library employed to document transactions over multiple computers so the recorded values can be changed using savings without changing the whole following blocks and without network cooperation. That enables members to study and audit cheap trades. Their authenticity is verified by collective collaboration through the sharing of their collective matters. The outcome is a strong workflow in which the uncertainty of participants regarding information safety is limited. Using a blockchain eliminates the definite feature of endless duplication of a digital asset. This feature allows any value unit to be carried just once and solves the long-term issue of dual costs. Blockchain is defined as an encryption valuation protocol. The exchange based on the blockchain is able to be performed quicker, extra securely, and more affordable than conventional systems. A blockchain is able to select title rights due to the fact that it provides a record that leads to presentation and acceptance. A distributed, consensus-based database chain that continuously maintains a list of records (categories) that each refer to previous list options, thereby strengthening it against unauthorized undermining or revisión[2,3]. The blockchain itself is a subset of Distributed Ledger technologies. Blockchain is a type of data architecture used in distributed office technology in which transaction records are stored in interconnected chains. In this technology, the network is able to maintain the integrity of the database content, despite the fact that multiple users record and modify data simultaneously and that data may overlap. Due to the encrypted data structure of the china block, the integrity is maintained without a central controller. There is a math problem that is difficult to solve in bitcoin distributed general ledgers to sort transactions and prevent inconsistencies, but after solving the problem, it is easy to use this mechanism, "proof of function" (Proof). of work) say[4,5]. In the bitcoin blockchain method, one 
can arrange the transactions of each step that has found the answer to this difficult question and at the same time the changes that it intends to apply (new block) do not contradict the previous steps of the chain. The method of detecting inconsistency is that the transactions of each block enter the hash function and everyone has the answer to that hash function. People can oppose unauthorized change without having to know which part has changed.

. What makes blockchain a highly efficient system for use in the medical system is information security, the impossibility of manipulating data to commit crimes such as drug abuse and illicit trafficking, and the transparency of data. Data has become the most important tool for any business. With technologies that can manage data on a large scale, both health care providers and patients, and of course, the insurance system, can quickly and efficiently advance their medical goals $[5,6,7]$.

The rapid adoption of digital technologies in healthcare resulted in the creation of huge volumes of digital patient reports. This expansion places unusual requirements on the protection of medical data in its usage and exchange. The development of blockchain (from now on referred to as BT) as a reliable and precise tool for storing and distributing data opens the way to new opportunities for solving serious problems of confidentiality, security, and data integrity in healthcare. Over the past few years, BT has attracted close attention from both industry and scientists. It is defined as a shared technology for peer to peer digital information network transactions that is able to be openly or personally shared to every users. $[8,9,10]$.

The interoperability purpose in healthcare is to aid the medical information exchange, including an Electronic Health Record (EHR), among patients and healthcare providers so this data is able to be distributed in an environment. [11]. Moreover, interoperability allows providers to securely exchange patient medical records (subject to patient approval), regardless of the provider's location or trust relationship [12]. This is especially important given that the sources of medical data are diverse. This aspect of interoperability is being addressed by BT, which has demonstrated the potential for secure storage, management, and sharing of data among the medical community [13].

In the healthcare sector, BT has a positive impact on healthcare outcomes for companies and concerned parties by streamlining business manners, improving patient results, managing data, improving regulatory compliance,

as well as decreasing expenses.[14].

The ability to share health data without compromising user privacy and data security is an important step towards making health care systems smarter and improving the quality of health care services and user experiences.

Currently, there is a significant amount of research that concerns the application of BT in healthcare, an exhaustive, extensive evaluative analysis of the current study on healthcare applications based on blockchain has not been reviewed. As a way of example, in the study performed by C. Ananth et al. [15] provides a brief overview of BT medical applications. The study addresses solely 3 fields: user-centred medicine, and drug counterfeiting, and public health management.

G. Drosatos and E. Kaldoudi [16] published the study on BT-based medical and biomedical applications. The study mainly discusses traditional BT (Bitcoin features) and its architecture. The authors then describe some aspects of BT for medical record management, insurance claim processes, and a medical data registry.

The study by J.M. Roman-Belmonte et al. [17] describes some of the uses of BT in health care. The use cases have especially focused on reconciling interoperability requirements and patient reports, and also supply chain management (SCM). The principal drawback of the research is the restriction of use problems as well as the lack of narrow tracking of new blockchain-based medical applications.

PTS. Liu [18] provides an analysis of BT medical applications. However, the study doesn't have access to the tests carried out in the investigated applications. In another study, W.J. Gordon and C. Catalini [19] investigated BT in health insurance, procurement processes, electronic health records, biomedical research. Furthermore, A.D. Rakic [20] studied numerous healthcare applications on the basis of blockchains, such as fraud detection, clinical examination, and EHR HS. Chen et al. [21] provided an overview of the healthcare industry's requirements for protecting patients' medical information using blockchain.

Based on this literature review, we believe that so far no study has comprehensively categorized BT in health applications from a health management perspective. To address this shortcoming, in this research, we intend to study various blockchain-based healthcare applications, with a focus on the latest developments in healthcare management. The purpose of the investigation is to investigate the prospects and risks of using BT in healthcare management.

The hypothesis of the study: BT will change the healthcare ecosystem, making not only a transparent and safe process for the provision of medical services, but also improving the quality of medical care, and at a lower cost.

According to the results of the study, it can be concluded that the goal set in the study has been achieved.

\section{Methods}

During the research, such research methods were applied as:

- analysis of scientific literature on the problem of the prospects for the use of BT in healthcare management; 
- expert survey. A number of questions were raised to the experts regarding the various current uses of BT for medical applications in health management; key challenges for medical applications in BT; guidelines for future research and open questions; advantages and disadvantages of current blockchain-based healthcare control uses.

Forty experts (employees of medical institutions, employees of IT companies) whose areas of interest include the application of BT in the healthcare sector took part in the expert online survey.

\section{Results}

According to experts, new healthcare technologies based on blockchain are structurally divided into 4 tiers: healthcare applications, information sources, BT, and parties concerned. Accordingly, the workflow of blockchain-based medical applications also consists of 4 central layers: raw medical records, BT, healthcare application, and parties concerned (Table 1).

Table 1: Workflow Framework for BT Medical Applications

\begin{tabular}{|l|l|l|}
\hline № & \multicolumn{1}{|c|}{ Level } & \multicolumn{1}{c|}{ Characteristic } \\
\hline 1 & Raw medical data & $\begin{array}{l}\text { First, the entire information from medical tools, labs, social media is connected } \\
\text { and generates basic information that is afterwards sized up to big information. } \\
\text { This information is a vital element of the complete blockchain-based } \\
\text { healthcare system. }\end{array}$ \\
\hline 2 & Blockchain technology & $\begin{array}{l}\text { Every blockchain platform holds various characteristics, including consensus } \\
\text { rules. Blockchain platforms encourage users to build and maintain their } \\
\text { activities. Numerous blockchain platforms are generated and applied, i.e., } \\
\text { Hyperledger Fabric and Ripple. } \\
3 \\
\text { Healthcare applications }\end{array}$ \\
\hline Healthcare applications & $\begin{array}{l}\text { based on the blockchain are able to be broken into 3 categories. Initially, data } \\
\text { management, such as universal scientific data exchange for EHR, research and } \\
\text { development (R\&D), data management. The next category is SCM } \\
\text { applications, such as clinical trials. The last class includes IoMT, such as the } \\
\text { blending of the Internet of Things (IoT) in medical devices, and AI. }\end{array}$ \\
\hline 4 & Parties concerned & $\begin{array}{l}\text { Parties concerned level is made up of parties that benefit from healthcare } \\
\text { applications based on blockchains, including people in business, and patients. } \\
\text { The central duty of users at this stage is to prepare and control information } \\
\text { minus jeopardizing their safety and privacy. }\end{array}$ \\
\hline
\end{tabular}

Note: compiled based on the expert survey.

According to the experts, with the development of electronic health-related data, cloud storage of medical data and rules for protecting the privacy of patient data, new possibilities are opening up for managing medical data, as well as for the convenience of patients when accessing and sharing their medical data. Securing data, storage, and transactions and managing their seamless integration are extremely valuable for any data-driven organization, especially in healthcare, where BT can solve these critical issues reliably and efficiently.

Based on the expert survey, seven stages of the workflow for managing health data in the blockchain were identified (Table 2).

\section{Table 2: Stages of Healthcare Data Management in the Blockchain}

\begin{tabular}{|c|l|}
\hline Stage & \multicolumn{1}{c|}{ Stage characteristics } \\
\hline I & Primary data is created as a result of the patient's interaction with their doctors and experts. \\
\hline II & $\begin{array}{l}\text { An electronic disease record is designed for every case applying the initial data obtained in the initial } \\
\text { level. }\end{array}$ \\
\hline III & $\begin{array}{l}\text { The individual patient owns a confidential electronic record, and private access is merely provided to } \\
\text { the property owner. Parties wishing to have access to this data have to demand confirmation from the } \\
\text { owner of the EHR, and the owner decides who will be granted access. }\end{array}$ \\
\hline IV & $\begin{array}{l}\text { These three stages are at the heart of the whole procedure, involving blockchain and database. The } \\
\text { database records in a divided way, and the blockchain supplies extraordinary privacy to guarantee } \\
\text { people, factual user access. }\end{array}$ \\
\hline V & \begin{tabular}{l} 
\\
\hline
\end{tabular}
\end{tabular}




\begin{tabular}{|c|l|}
\hline VI & \\
\hline VII & $\begin{array}{l}\text { Healthcare providers (clinics, medical centers, hospitals) are the end user who, with the permission of } \\
\text { the owner, gains access to safe and reliable services. Regardless of the patient's location, their EHR will } \\
\text { be available, including on the phone, and confirmed using a distributed ledger (blockchain), to which } \\
\text { healthcare providers can continue to add data. }\end{array}$ \\
\hline
\end{tabular}

Note: compiled based on the expert survey.

Blockchain applications in the healthcare data management category include the following capabilities (Table 3).

Table 3: BT. Opportunities in the Health Data Management Category

\begin{tabular}{|l|l|c|}
\hline № & \multicolumn{1}{|c|}{ BT. Opportunities } & $\% *$ \\
\hline 1 & Data sharing & $75 \%$ \\
\hline 2 & Data management & $80 \%$ \\
\hline 3 & Data storage (e.g. cloud applications) & $65 \%$ \\
\hline 4 & Electronic health record & $85 \%$ \\
\hline
\end{tabular}

Note: compiled based on the expert survey; * - percentage of expert references.

Further, we'll discuss BT opportunities in the health data management category in more detail.

\section{Discussion}

Data sharing. According to the experts, the distribution of medical and health data is one of the primary and significant steps towards improving the medical services quality and a smart health system. The exchange of medical records can take place between people, for example, a case who needs to share their medical records with the expert at the initial encounter. Besides, the exchange can take place between a person and an interested party, for example, a patient who shares their records with an insurance

firm. Yet information can be transferred abroad.

Foreign scientists have studied the

aspect of using blockchain-based applications. Thus, H. Joudaki et al. [22] proposed a registration system to promote the trade of digital health information among nations in the safest way, utilizing a special blockchain. G. Bordea et al. [23] considered various applications for the exchange of medical data, given the architecture of the blockchain. The proposed solution helps to control and exchange customer data without compromising confidentiality easily.

The study [24] developed the MedBlock framework based on BT to solve the problem of data management and data sharing in the EHR system and improve the exchange of medical information. Patients can access the EHRs of different hospitals through the MedBlock structure, avoiding segmentation of previous medical data into different databases. In addition, data sharing and collaboration across the blockchain can help hospitals gain an early understanding of a patient.

Data management. The experts note that even though many medical institutions manage data, the volume of which is constantly growing, the security and confidentiality of data are constantly violated, both unintentionally and by illegal users. As a result, healthcare facilities may experience a loss of reputation. Different data users have different roles, and access to data should be governed by the privileges assigned to those roles. This access mode can be provided using BT.

As an example, here are some BT that have been developed for this purpose.

MedRec [25] is a decentralized EHR management system in which data access rights and transactions are recorded on the blockchain, and execution is completed by smart contracts. MedRec aggregates the complete medical information of a healthcare provider for authentication, privacy, auditing, and data exchange and provides patients with comprehensive, immutable medical data and services. Another study [26,27] presented a new idea for changing consent management in the healthcare system, which provides the user with the ability to control all medical record data using the blockchain

\section{Electronic health record.}

Information technology has provided an opportunity to facilitate the work of medical workers through the introduction of electronic medical records.

According to the experts ( $85 \%$ of respondents), electronic access to medical records has allowed doctors to significantly improve the quality of treatment. In addition, EHR allows for better disease control and increased levels of preventive care, while digital recording enables improved decision support functions and greater collaboration between caregivers[28,29]. 
Much research work has focused on the development of BT to protect, exchange and store EHR data both within and between institutions. Y. Chen et al. [29] developed a secure blockchain structure for the exchange of medical data by designing secure cloud storage for confidential patient medical records. In this structure, the management of medical data is achieved through a digital archive, which has the right to control access to the information of its owners. This is maintained by deploying cloud encryption on-chain. In the study by R. Guo et al. [30] presented an attribute-based signature scheme using multi-authorization BT to guarantee and verify electronic records. It facilitates multicast messaging and can resist hacker attacks [31].

\section{Conclusion}

$\mathrm{BT}$ is attracting important consideration from people and companies of approximately all classes and sizes. It is able to transform the old industry through its functions, which involve anonymity, decentralization, consistency. It is redefining the modelling of the data utilized in various medical purposes. This is essentially because of its flexibility and the capability to segment, preserve and share medical information and aids in unique ways. It is at the core of several continuing improvements in the healthcare system.

In this research, we examined multiple blockchains uses in the healthcare industry and recognized fundamental investigation initiatives and future study possibilities. Particularly, we showed the abilities of BT for handling health data and how blockchain can permit patients and facilitate the sharing of health data procedure. We discovered that there remains an agreement between specialists that along with BT, the patient document can genuinely be maintained and managed by the legitimate owner of the data, such as the patient. Blockchain enables time-stamping medical records so that no one can tamper with them. Patients will be entitled to determine who can have access to their information and why.

Thus, the hypothesis of the study that BT will change the healthcare ecosystem was confirmed, making not only a transparent and safe process for the provision of medical services but also improving the quality of medical services and at a lower cost.

However, there are still several issues that require further study. Therefore, further research can be devoted to the application of blockchain in the areas of SCM and IoMT in healthcare, in particular, the applicability of blockchain for solving the problem of degradation of trust and increasing the transparency of data on clinical trials.

\section{References}

[1] Nofer, M. et al. (2017). Blockchain. Business \& Information Systems Engineering, 59(3), 183-187.

[2] Cohn, A., West, T., \& Parker, C. (2017). Smart after all: blockchain, smart contracts, parametric insurance, and smart energy grids. Georgetown Law Technology Review, 1(2), 273-304.

[3] Deeva, T.V., Nikiporets-Takigawa, G., Lustina, T.N., Podsevalova, E.N., \& Didenko, E.N. (2020). Blockchain Technologies and Smart Contracts: New Technological Methods to Regulate Transactions and Trade Operations. International Journal of Emerging Trends in Engineering Research, 8(7), 3659-3664.

[4] Yangutov, L.E., Abaeva, L.L., \& Abaev, N.W. (2020). The cult of the heaven in the religious tradition of peoples of Eastern and Inner Asia. Revista Inclusiones, 7 num Especial, 493-508.

[5] Glembotskaya, G.T., Eremin, S.Yu., \& Chupandina, E.E. (2020). Scientific priorities and real prospects for cost optimization in formulation development. Entrepreneurship and Sustainability Issues, 7(3), 1484-1499.

[6] Garankina, R.Yu., Zakharochkina, E.R., Samoshchenkova, I.F., Kachmarskaya, L.M., \& Lebedev, A.V. (2019). Marketing analysis of the required drugs in pharmacies. $J$ Adv Pharm Edu Res, 9(4), 76-82.

[7] Dunphy, P., \& Petitcolas, F.A. (2018). A first look at identity management schemes on the blockchain. IEEE Security \& Privacy, 16(4), 20-29.

[8] Dorri, A., Steger, M., Kanhere, S.S., \& Jurdak, R. (2017). Blockchain: A distributed solution to automotive security and privacy. IEEE Communications Magazine, 55(12), 119-125.

[9] Kuo, T-T., Kim, H-E., \& Ohno-Machado, L. (2017). Blockchain distributed ledger technologies for biomedical and health care applications. Journal of the American Medical Informatics Association, 24(6), 1211-1220.

[10] Randall, D., Goel, P., \& Abujamra, R. (2017). Blockchain Applications and Use Cases in Health Information Technology. Journal of Health \& Medical Informatics, 8(3), 8-11.

[11] Tak, P., \& Liu, S. (2016). Medical record system using blockchain, big data and tokenization. International Conference on Information and Communications Security. Springer; 254-261.

[12] Dubovitskaya, A., Xu, Z., Ryu, S., Schumacher, M., \& Wang, F. (2017). Secure and Trustable Electronic Medical Records Sharing using blockchain. AMIA Аnnu Symp Proc, 16, 650-659.

[13] Iansiti, M., \& Lakhani, K.R. (2017). The truth about blockchain. Harvard Business Review, 95(1), 118-127.

[14] Yoon, H.J. (2019). Blockchain technology and healthcare. Healthcare Informatics Research, 25(2), 59-60. 
[15] Ananth, C., Karthikeyan, M., \& Mohananthini, N. (2018). A secured healthcare system using private blockchain technology. Journal of Engineering Technology, 6, 42-54.

[16] Drosatos, G., \& Kaldoudi, E. (2019). Blockchain applications in the biomedical domain: a scoping review. Computational and Structural Biotechnology Journal, 17, 229-240.

[17] Roman-Belmonte, J.M., De la Corte-Rodriguez, H., \& Rodriguez-Merchan, E.C. (2018). How blockchain technology can change medicine. Postgraduate Medical Journal, 130(4), 420-427

[18] Liu, P.T.S. (2016). Medical record system using blockchain, big data and tokenization. Lam K-Y., Chi C-H., Qing S. (eds.). Information and communications security. ICICS 2016. Lecture notes in computer science. Springer; 254-261.

[19] Gordon, W.J., \& Catalini, C. (2018). Blockchain Technology for Healthcare: Facilitating the Transition to Patient-Driven Interoperability. Computational and Structural Biotechnology Journal, 16, 224-230.

[20] Rakic, D. (2018). Blockchain Technology in Healthcare. Proceedings of the 4th International Conference on Information and Communication Technologies for Ageing Well and e-Health, 13-20

[21] Chen, H.S., Jarrell, J.T., Carpenter, K.A., Cohen, D.S., \& Huang, X. (2019). Blockchain in Healthcare: A Patient-Centered Model. Biomedical Journal of Scientific \& Technical Research, 20(3), 15017-15022.

[22] Joudaki, H. et al. (2014). Using data mining to detect health care fraud and abuse: a review of literature. Global Journal of Health Science, 7(1), 194-202.

[23] Bordea, G., Jothi, N., Rashid, N.A., \& Husain, W. (2015). Data mining in healthcare: a review. Procedia Computer Science, 72, 306-313.

[24] Fan, K., Wang, S., Ren, Y., Li, H., \& Yang, Y. (2018). Medblock: Efficient and secure medical data sharing via blockchain. The Journal of Medical Systems, 42: 136.

[25] Azaria, A., Ekblaw, A., Vieira, T., \& Lippman, A. (2016). Medrec: Using blockchain for medical data access and permission management. Proceedings of the 2016 2nd International Conference on Open and Big Data (OBD). Vienna, 25-30.

[26] Xia, Q. et al. (2017). MeDShare: Trust-less medical data sharing among cloud service providers via blockchain. IEEE Access, 5, 14757-14767.

[27] Al Omar, A. et al. (2019). Privacy-friendly platform for healthcare data in cloud based on blockchain environment. Future Generation Computing Systems, 95, 511-521.

[28] Kaur, H. et al. (2018). A Proposed Solution and Future Direction for Blockchain-Based Heterogeneous Medicare Data in Cloud Environment. The Journal of Medical Systems, 42, 156-165.

[29] Chen, Y. et al. (2018). Blockchain-Based Medical Records Secure Storage and Medical Service Framework. The Journal of Medical Systems, 43, 5-15.

[30] Guo, R., Shi, H., Zhao, Q., \& Zheng, D. (2018). Secure attribute-based signature scheme with multiple authorities for blockchain in electronic health records systems. IEEE Access, 776: 1-12.

[31] Babaskin, D.V., Litvinova, T.M., Babaskina, L.I., Ovakimyan, A.K., \& Kolevatova, K.Y. (2020). Marketing Evaluation of Consumer Preferences in Using Mobile Apps for Healthcare to Support Drug Adherence. Periódico Tchê Química, 17(35), 1013-1027. 